\title{
AN EXTENSION OF RENEWAL THEORY
}

\section{KAI LAI CHUNG ${ }^{1}$ AND HARRY POLLARD}

Renewal theory can be and has been viewed in several different ways. One way is to reduce the problems to those concerning the addition of independent, non-negative random variables having a common distribution. Accordingly we introduce the random variables $X_{1}, X_{2}, \cdots$, independent, non-negative, and all possessing the same distribution function $F(x)$ with mean $m=\int_{-\infty}^{\infty} x d F(x) \quad(0<m$ $\leqq \infty)$, and their successive sums $S_{n}=\sum_{k=1}^{n} X_{k}$. Either $F(x)$ is purely discontinuous with all its discontinuities located at the multiples of a fixed real number or it is not so; we call the first case the lattice case and the second the nonlattice case. In the lattice case there is no real loss of generality by assuming (as we shall do in the following) the said discontinuities are all located at integers whose greatest common divisor is one. One of the main results of renewal theory can then be stated as follows:

(i) In the lattice case, if $x$ runs through integers:

$$
\lim _{x \rightarrow \infty} \sum_{n=1}^{\infty} P\left(S_{n}=x\right)=\frac{1}{m}
$$

(Erdös, Feller, and Pollard [1]; an equivalent theorem had been given by Kolmogorov [2] previously).

(ii) In the nonlattice case, if $h$ is any positive number:

$$
\lim _{x \rightarrow \infty} \sum_{n=1}^{\infty} P\left(x \leqq S_{n} \leqq x+h\right)=\frac{h}{m}
$$

(Doob [3], Blackwell [4]). In both cases $1 / m=0$ if $m=\infty$.

In renewal theory only non-negative random variables are considered but the formulas (1) and (2) remain meaningful if we drop the assumption of non-negativeness. In other words, it is legitimate to inquire whether they are still true when the random variables are allowed to assume both positive and negative values, but otherwise subject to the same conditions as before. Our answer to this question is affirmative, but not without some restrictions which we believe to be unnecessary but which we are unable to remove at present. ${ }^{2}$ To be precise, we can prove the following:

Received by the editors February 8, 1951 and, in revised form, July 20, 1951.

${ }^{1}$ Research done under contract with the Office of Naval Research.

${ }^{2}$ Added in proof. In the meantime T. E. Harris and D. Blackwell informed us that they had removed these restrictions. Their methods are entirely different from ours. 
(i) In the lattice case, if $x$ runs through integers and $m \neq \pm \infty$, then

$$
\lim _{x \rightarrow \infty} \sum_{n=1}^{\infty} P\left(S_{n}=x\right)=\left\{\begin{array}{cll}
1 / m & \text { if } & m>0, \\
0 & \text { if } & m<0 .
\end{array}\right.
$$

(ii) In the nonlattice case, if $h$ is any positive number and if we assume in addition that $\lim \sup _{t \rightarrow \infty}|f(t)|<1$ where $f(t)=\int_{-\infty}^{\infty} e^{i t x} d F(x)$, and $m \neq \pm \infty$, then

$$
\lim _{x \rightarrow \infty} \sum_{n=1}^{\infty} P\left(x \leqq S_{n} \leqq x+h\right)=\left\{\begin{array}{clc}
h / m & \text { if } & m>0 \\
0 & \text { if } & m<0 .
\end{array}\right.
$$

Analogies for $x \rightarrow-\infty$ are obvious. The additional assumption made in (ii) is satisfied if for example $F(x)$ contains a nonvanishing absolutely continuous part. (This was Doob's original assumption about (2) later removed by Blackwell.) It should be mentioned that if $m=0$, then both series in (3) and (4) diverge for every integral and real $x$, respectively; see [5].

In the following we shall prove only (4), for two reasons. First, the proof of (3) is entirely similar and indeed simpler; second, another simpler proof of (3) has subsequently been found by Chung and Wolfowitz which will appear elsewhere [6].

One more remark: The method we use, that of Fourier inversion and direct calculation, seems new in this connection. It is suggested by a similar approach to another, not unrelated, problem employed by Chung and Fuchs [5]. The actual calculation, however, turns out to be quite different.

$1^{\circ}$. To prove (4) it is sufficient to prove that

(5) $\lim _{x \rightarrow \infty} \lim _{r \rightarrow 1-0} \sum_{n=1}^{\infty} r^{n} \int_{0}^{h} P\left(\left|S_{n}-x\right| \leqq u\right) d u=\left\{\begin{array}{rll}h^{2} / m & \text { if } & m>0, \\ 0 & \text { if } & m<0 .\end{array}\right.$

For, the existence of the inner limit implies that

$$
L(x, u)=\sum_{n=1}^{\infty} P\left(\left|S_{n}-x\right| \leqq u\right)<\infty
$$

for all $x$ and $u$, and moreover that

$$
\lim _{x \rightarrow \infty} \int_{0}^{h} L(x, u) d u=\left\{\begin{array}{clc}
h^{2} / m & \text { if } & m>0, \\
0 & \text { if } & m<0 .
\end{array}\right.
$$

Suppose $m>0$. If $0<h_{1}<h_{2}$, since $L(x, u)$ is nondecreasing in $u$,

$$
L\left(x, h_{1}\right)\left(h_{2}-h_{1}\right) \leqq \int_{h_{1}}^{h_{2}} L(x, u) d u \leqq L\left(x, h_{2}\right)\left(h_{2}-h_{1}\right) .
$$


Letting $x \rightarrow \infty$ we obtain, using (6),

$$
\limsup _{x \rightarrow \infty} L\left(x, h_{1}\right) \leqq \frac{h_{1}+h_{2}}{m} \geqq \liminf _{x \rightarrow \infty} L\left(x, h_{2}\right) .
$$

Letting $h_{2} \downarrow h_{1}$ in the first and $h_{1} \uparrow h_{2}$ in the second inequality we obtain

$$
\limsup _{x \rightarrow \infty} L\left(x, h_{1}\right) \leqq \frac{2 h_{1}}{m}, \quad \liminf _{x \rightarrow \infty} L\left(x, h_{2}\right) \geqq \frac{2 h_{2}}{m} .
$$

Hence $\lim _{x \rightarrow \infty} L(x, h)=2 h / m$ which is equivalent to (4). The case $m<0$ is even simpler.

$2^{\circ}$. Using a well known Fourier inversion formula we can write the sum in (5) as

$$
\begin{gathered}
\sum_{n=0}^{\infty} r^{n} \frac{1}{\pi} \int_{-\infty}^{\infty} e^{-i t x} \frac{1-\cos h t}{t^{2}}(f(t))^{n} d t \\
\quad=\frac{1}{\pi} \int_{-\infty}^{\infty} \frac{1-\cos h t}{t^{2}} \frac{e^{-i t x}}{1-r f(t)} d t
\end{gathered}
$$

if $0<r<1$ and $x>h$, the term corresponding to $n=0$ vanishing. We wish to evaluate

$$
\lim _{x \rightarrow+\infty} \lim _{r \rightarrow 1-0} \frac{1}{\pi} \int_{-\infty}^{\infty} \frac{1-\cos h t}{t^{2}} \frac{e^{-i t x}}{1-r f(t)} d t .
$$

$3^{\circ}$. To do this we first show that for any $\delta>0$

$$
\lim _{x \rightarrow \infty} \lim _{r \rightarrow 1-0} \int_{|t| \geqq \delta} \frac{1-\cos h t}{t^{2}} \frac{e^{-i t x}}{1-r f(t)} d t=0 .
$$

Since $\lim \sup _{t \rightarrow \infty}|f(t)|<1$ we have $\sup _{|t| \geq \delta}|f(t)|=\epsilon(\delta)<1$. Thus we can take the limit with respect to $r$ inside the integral sign in (9). (9) then follows by the Riemann-Lebesgue lemma in Fourier analysis.

Let $\epsilon=(1-r) / r$, then $1-r f(t)=r\{\epsilon+[1-f(t)]\}$. Taking the real part of (8), as we plainly may, and confining ourselves with the range $|t|<\delta$, on account of (9), we must now show that

$$
\begin{gathered}
\lim _{\delta \rightarrow 0} \lim _{x \rightarrow \infty} \lim _{\epsilon \rightarrow 0} \frac{1}{\pi} \int_{|t|<\delta} \frac{1-\cos h t}{t^{2}} R\left\{\frac{e^{-i t x}}{\epsilon+1-f(t)}\right\} d t \\
=\frac{h^{2}}{2}\left(\frac{1}{m}+\frac{1}{|m|}\right) .
\end{gathered}
$$

The extra limit in $\delta$ is added for the sake of convenience. 
Now we have

$$
\begin{aligned}
1+i m t-f(t)= & \int_{-\infty}^{\infty}(1-\cos t x) d F(x) \\
& +i \int_{-\infty}^{\infty}(t x-\sin t x) d F(x) .
\end{aligned}
$$

Let

$$
\begin{aligned}
& R=R(t)=\int_{-\infty}^{\infty}(1-\cos t x) d F(x) \\
& I=I(t)=\int_{-\infty}^{\infty}(t x-\sin t x) d F(x)
\end{aligned}
$$

Then $R(t) \geqq 0, R(t)$ is even and $I(t)$ is odd. We have

$$
R\left\{\frac{e^{-i t x}}{\epsilon+1-f(t)}\right\}=\frac{(\epsilon+R) \cos t x+(m t-I) \sin t x}{(\epsilon+R)^{2}+(m t-I)^{2}} .
$$

$4^{\circ}$. Consider

$$
\lim _{x \rightarrow \infty} \lim _{\epsilon \rightarrow \infty} \frac{1}{\pi} \int_{|t|<\delta} \frac{1-\cos h t}{t^{2}} \frac{(m t-I) t}{(\epsilon+R)^{2}+(m t-I)^{2}} \frac{\sin t x}{t} d t .
$$

Since $R(t) \geqq 0$ and $R(t)=o(t), I(t)=o(t)$ as $t \rightarrow 0$, we have

$$
\frac{|(m t-I) t|}{(\epsilon+R)^{2}+(m t-I)^{2}} \leqq \frac{C_{1} t^{2}}{R^{2}+(m t-I)^{2}} \leqq C_{2}
$$

where $C_{1}, C_{2}$, and so forth denote absolute constants. Hence we can first let $\epsilon \rightarrow 0$ under the integral sign in (11); then let $x \rightarrow \infty$ and we get, by standard argument, as the value of (11):

$$
\lim _{t \rightarrow 0} \frac{1-\cos h t}{t^{2}} \frac{(m t-I) t}{R^{2}+(m t-I)^{2}}=\frac{h^{2}}{2 m} .
$$

Thus in order to prove (10) it remains to show that

$$
\begin{aligned}
& \lim _{\delta \rightarrow 0} \lim _{x \rightarrow \infty} \lim _{\epsilon \rightarrow 0} \frac{1}{\pi} \int_{|t|<\delta} \frac{1-\cos h t}{t^{2}} \frac{(\epsilon+R) \cos t x}{(\epsilon+R)^{2}+(m t-I)^{2}} d t \\
& =\frac{h^{2}}{2|m|} \text {. }
\end{aligned}
$$

Let 


$$
H=H(t)=(1-\cos h t) / t^{2}, \quad A=A(t)=m t-I(t) .
$$

The integral in (12) can be written as

$$
H \frac{(\epsilon+R)(\cos t x-1)}{(\epsilon+R)^{2}+A^{2}}+H \frac{\epsilon}{(\epsilon+R)^{2}+A^{2}}+H \frac{R}{(\epsilon+R)^{2}+A^{2}} .
$$

Call these three parts $J_{1}, J_{2}$, and $J_{3}$ respectively.

We need the following lemma.

Lemma. $R /\left(R^{2}+A^{2}\right)$ is integrable in $|t|<\delta$.

Proof. Since $A^{2} \sim m^{2} t^{2}$ as $t \rightarrow 0$, we have

$$
\frac{R}{R^{2}+A^{2}} \leqq C_{3} \frac{R}{t^{2}} \text {. }
$$

By definition we have

$$
\int_{|t|<\delta} \frac{R}{t^{2}} d t=\int_{-\delta}^{\delta} \frac{d t}{t^{2}} \int_{-\infty}^{\infty}(1-\cos t x) d F(x) .
$$

Since the integrand is positive, we can invert the double integral and get

$$
\begin{aligned}
\int_{-\infty}^{\infty} d F(x) \int_{-\delta}^{\delta} \frac{1-\cos t x}{t^{2}} d t & =\int_{-\infty}^{\infty}|x| d F(x) \int_{-\delta|x|}^{\delta|x|} \frac{1-\cos t}{t^{2}} d t \\
& \leqq \int_{-\infty}^{\infty}|x| d F(x) \int_{-\infty}^{\infty} \frac{1-\cos t}{t^{2}} d t<\infty .
\end{aligned}
$$

$5^{\circ}$. We have, by the lemma,

$$
\begin{aligned}
\lim _{\delta \rightarrow 0} \lim _{x \rightarrow \infty} \lim _{\epsilon \rightarrow 0} \int_{|t|<\delta} J_{3} d t & =\lim _{\delta \rightarrow 0} \lim _{\epsilon \rightarrow 0} \int_{|t|<\delta} H \frac{R}{(\epsilon+R)^{2}+A^{2}} d t \\
& =\lim _{\delta \rightarrow 0} \int_{|t|<\delta} H \frac{R}{R^{2}+A^{2}} d t=0 .
\end{aligned}
$$

Next, we notice that

$$
\frac{|\cos t x-1|}{(\epsilon+R)^{2}+A^{2}} \leqq \frac{C_{4} t^{2}}{R^{2}+A^{2}} \leqq C_{5}
$$

for fixed $x$ and $|t|<\delta$. Hence

$$
\lim _{R \rightarrow 0} \int_{|t|<\delta} J_{1} d t=\int_{|t|<\delta} H \frac{(\cos t x-1) R}{R^{2}+A^{2}} d t
$$


Now let $x \rightarrow \infty$, by the Riemann-Lebesgue lemma and the lemma above we get as limit

$$
\int_{|t|<\delta} H \frac{-R}{R^{2}+A^{2}} d t
$$

Finally let $\delta \rightarrow 0$ and we get 0 . Hence

$$
\lim _{\delta \rightarrow 0} \lim _{x \rightarrow \infty} \lim _{\epsilon \rightarrow 0} \int_{|t|<\delta} J_{1} d t=0 .
$$

It remains to consider $J_{2}$ and to show that

$$
\lim _{\delta \rightarrow 0} \lim _{\epsilon \rightarrow 0} \frac{1}{\pi} \int_{|t|<\delta} H \frac{\epsilon}{(\epsilon+R)^{2}+A^{2}} d t=\frac{h^{2}}{2|m|} .
$$

By standard argument we have

$$
\lim _{\delta \rightarrow 0} \lim _{\epsilon \rightarrow 0} \frac{1}{\pi} \int_{|t|<\delta} H \frac{\epsilon}{\epsilon^{2}+m^{2} t^{2}} d t=\frac{h^{2}}{2|m|} .
$$

Therefore we must show that

(16) $\lim _{\delta \rightarrow 0} \lim _{\epsilon \rightarrow 0} \frac{1}{\pi} \int_{|t|<\delta} H\left\{\frac{\epsilon}{\epsilon^{2}+m^{2} t^{2}}-\frac{\epsilon}{(\epsilon+R)^{2}+A^{2}}\right\} d t=0$.

$6^{\circ}$. The expression in brackets in (16) is

$$
\frac{\epsilon\left(R^{2}+A^{2}-m^{2} t^{2}\right)+2 \epsilon^{2} R}{\left(\epsilon^{2}+m^{2} t^{2}\right)\left[(\epsilon+R)^{2}+A^{2}\right]} .
$$

Consider the first term and the corresponding integral:

$$
\begin{aligned}
\int_{|t|<\delta} H \frac{\epsilon\left(R^{2}+A^{2}-m^{2} t^{2}\right)}{\left(\epsilon^{2}+m^{2} t^{2}\right)\left[(\epsilon+R)^{2}+A^{2}\right]} d t & \\
\leqq & \int_{|t|<\delta} H \frac{\epsilon}{\epsilon^{2}+m^{2} t^{2}} \frac{\left|R^{2}+A^{2}-m^{2} t^{2}\right|}{R^{2}+A^{2}} d t
\end{aligned}
$$

By standard argument the limit of the last integral $\delta \rightarrow 0$ is

$$
\lim _{t \rightarrow 0} B \frac{\left|R^{2}+A^{2}-m^{2} t^{2}\right|}{R^{2}+A^{2}}=0 .
$$

Next, consider the second term and the corresponding integral:

$$
\int_{|t|<\delta} H \frac{2 \epsilon^{2} R}{\left(\epsilon^{2}+m^{2} t^{2}\right)\left[(\epsilon+R)^{2}+A^{2}\right]} d t .
$$


The integrand is dominated by $2 R H /\left(R^{2}+A^{2}\right)$ which is integrable in $|t|<\delta$ by the lemma, hence we may let $\epsilon \rightarrow 0$ under the integral sign and get 0 as limit.

(13)-(16) establish (12), whence the desired result.

\section{REFERENCES}

1. P. Erdös, W. Feller, and H. Pollard, A theorem on power series, Bull. Amer. Math. Soc. vol. 55 (1949) pp. 201-204.

2. A. Kolmogorov, Anfangsgrunde der Theorie der Markoffschen Ketten mit unendlichen vielen möglichen Zuständen, Rec. Math. (Mat. Sbornik) N.S. vol. 1 (1936) pp. 607-617; the proofs are contained in another paper in Russian: Bulletin de l'Universite d'Etat à Moscou (A) vol. 1 (1937) pp. 1-15.

3. J. L. Doob, Renewal theory from the point of view of the theory of probability, Trans. Amer. Math. Soc. vol. 63 (1948) pp. 422-438.

4. D. Blackwell, A renewal theorem, Duke Math. J. vol. 15 (1948) pp. 145-151.

5. K. L. Chung and W. H. J. Fuchs, On the distribution of values of sums of random variables, Memoirs of the American Mathematical Society, no. 6.

6. K. L. Chung and J. Wolfowitz, On a limit theorem in renewal theory, Ann. of Math. vol. 55 (1952) pp. 1-6.

Columbia University and Cornell University 